\title{
A Novel DLG3 Mutation Expanding the Phenotype of X-Linked Intellectual Disability Caused by DLG3 Nonsense Variants
}

\author{
Anna Sandestig ${ }^{a} \quad$ Anna Green ${ }^{a} \quad J$ Johan Aronsson ${ }^{b}$ Katarina Ellnebo ${ }^{a}$ \\ Margarita Stefanova ${ }^{a}$ \\ ${ }^{a}$ Department of Clinical Genetics, University Hospital Linköping, Linköping, Sweden; ${ }^{\text {b } D e p a r t m e n t ~ o f ~ P e d i a t r i c s, ~}$ \\ Ryhov County Hospital, Jönköping, Sweden
}

\section{Established Facts}

- Disease-causing mutations in DLG3 have been associated with a moderate to severe nonsyndromic Xlinked mental retardation (OMIM 300850). No constant dysmorphic features were noted.

\section{Novel Insights}

- A disease-causing mutation in the DLG3 gene has been found in a boy with marked facial dysmorphisms, including features not previously described.

- Even short stature due to growth hormone deficiency is unique to the boy presented here. This observation broadens the clinical spectrum of DLG3 nonsense mutations and raises the question whether nonsyndromic $\mathrm{X}$-linked mental retardation is appropriate.

\section{Keywords}

Clinical heterogeneity . DLG3 nonsense mutation .

Dysmorphology · Exome sequencing · Facial

dysmorphology $\cdot$ X-linked intellectual disability

\begin{abstract}
The DLG3 gene is located at Xq13.1 and encodes SAP102, a member of the MAGUK protein family, extensively expressed in the brain and involved in synaptic function. Mutations in DLG3 are associated with a rare nonsyndromic form of $\mathrm{X}$ linked intellectual disability (XLID) and have been described in 11 families to date. All affected males presented with intellectual disability, and some showed additional clinical fea-
\end{abstract}

\section{KARGER}

(c) 2019 S. Karger AG, Basel

E-Mail karger@karger.com

www.karger.com/msy tures. The majority of female carriers were reported asymptomatic or mildly affected, due to skewed X-inactivation, rarely severely affected. We report a family, a boy and his mother, with a novel nonsense mutation in the DLG3 gene, c.1720C>T; p.Arg574*. The boy, hemizygous for the variant, showed intellectual disability, short stature due to growth hormone deficiency, dysmorphic features, and pectus excavatum. The mother, who presented with learning disabilities and borderline cognitive development, is a heterozygous carrier of the variant, which had arisen de novo. X-inactivation test was noninformative. This case report broadens the phenotypic spectrum of XLID caused by DLG3 nonsense variants. The dysmorphic features of the affected males may be more frequent than previously thought.

(C) 2019 S. Karger AG, Basel

Margarita Stefanova

Department of Clinical Genetics

University Hospital Linköping

SE-58185 Linköping (Sweden)

E-Mail margarita.stefanova@ regionostergotland.se 
The human DLG3 (discs large homolog 3; OMIM *300189) gene is located at Xq13.1 and encodes synapseassociated protein 102 (SAP102), a member of the membrane-associated guanylate kinase (MAGUK) protein family [Zanni et al., 2010]. MAGUKs are scaffolding proteins for ionotropic glutamate receptors at excitatory synapses [Sheng and Hoogenraad, 2007]. They interact with a wide variety of proteins including receptors, adhesion, cytoskeletal and signaling molecules, and serve an important role in structural and functional synaptic plasticity thereby influencing dendritic spine stabilization and receptor localization [Funke et al., 2005; Elias and Nicoll, 2007]. SAP102 is the dominant scaffold in the fetal and neonatal brain and is associated with glutamate receptor targeting to synapses during neuronal development $[\mathrm{Mu}-$ rata and Constantine-Paton, 2013; Won et al., 2017]. MAGUKs, including SAP102, are composed of $3 \mathrm{com}-$ mon signaling domains, PDZ domains (abreviation of the first letters of the first three proteins discovered to share the domain) [Kennedy, 1995], followed by a SH3 domain (Src-homology-3), and a GK domain (guanylate kinase) [Müller et al., 1996]. Whereas the PDZ domains bind to cell adhesion molecules, ion channels, and receptors, the $\mathrm{SH} 3$ and GK domains interact with cytoskeletal proteins and intracellular signaling complexes [Müller et al., 1996; Won et al., 2017].

Mutations in DLG3 have been reported mainly as a rare cause of nonsyndromic X-linked intellectual disability (XLID) (MRX90; OMIM 300850) only in 11 families to date [Tarpey et al., 2004; Zanni et al., 2010; Isrie et al., 2012; Philips et al., 2014; Tzschach et al., 2015; Kumar et al., 2016], and they are summarized by Gieldon et al. [2017]. All affected males presented with cognitive impairment, ranging from mild to severe, and some showed additional clinical features such as seizures, abnormal behavior, and discrete facial dysmorphisms [Gieldon et al., 2017]. The majority of female carriers is reported asymptomatic or mildly affected. However, Gieldon et al. [2017] report a female carrier as severely affected as males due to skewed X-inactivation - the normal DLG3 allele was inactive. They showed that the severity of phenotypic expression in female carriers correlates with the $\mathrm{X}$-inactivation status.

We report a family, a boy and his mother, with a nonsense mutation in the DLG3 gene, c.1720C>T; p.Arg574*. The boy showed intellectual disability (ID), an abnormal behavior, short stature due to growth hormone (GH) deficiency, and dysmorphic features as well as divergent strabismus and pectus excavatum. His mother presented with learning difficulties at school, borderline cognitive development, and no dysmorphic features. She is a heterozygous carrier of the variant, which had arisen de novo.

\section{Clinical Report}

The patient, a 9-year-old boy, is the first child to nonconsanguineous parents of Swedish origin. He was born after an uneventful pregnancy after 42 weeks' gestation with the following birth parameters: weight $3,535 \mathrm{~g}( \pm 0 \mathrm{SD})$, length $52 \mathrm{~cm}(+0.5 \mathrm{SD})$, and head circumference $34 \mathrm{~cm}(-1 \mathrm{SD})$. Difficulties with breast feeding and mild generalized hypotonia were reported in the neonatal period.

The boy presented with moderate ID, an attention deficit, and hyperactive behavior as well as a distinct outgoing personality. $\mathrm{He}$ first learned to walk at the age of 3 years and 2 months. He had delayed speech development. At the age of 4.5 years, he only spoke 4 words, and at 8 years of age, he only rarely had spoken 3 -word phrases. The psychological evaluation at the age of 6 years assessed his development to be corresponding to the age level of 3 or 4 years. Postnatally, he developed a short stature due to GH deficiency, with a good response to GH therapy. Low serum levels of insulinlike growth factor 1 (IGF1), between 6 and $14 \mu \mathrm{g} / \mathrm{L}$ (reference levels for his age/sex 22-204 $\mu \mathrm{g} / \mathrm{L}$ ), as well as low serum levels of insulin-like growth factor-binding protein 3 (IGFBP3), between 1.141 and $1.619 \mu \mathrm{g} / \mathrm{L}$ (reference levels for his age/sex 1.719-4.959 $\mu \mathrm{g} / \mathrm{L}$ ), were measured between the age of 2.6 and 3 years. His height at the age of 2.5 years was $84 \mathrm{~cm}(-2.5 \mathrm{SD})$, and his weight was 10 $\mathrm{kg}(-3 \mathrm{SD})$. Head circumference at 3 years of age was $49 \mathrm{~cm}(-1.5$ SD). GH therapy was initiated at the age of 3.5 years, and his height increased from -2.5 to $-1 \mathrm{SD}$ at 6 years. Weight and height at the age of 7 years and 9 months were $24 \mathrm{~kg}(-1 \mathrm{SD})$ and $123 \mathrm{~cm}(-1.5$ $\mathrm{SD})$, respectively.

Dysmorphic features included: a triangular face, frontal bossing, prominent supraorbital ridges, downslanting palpebral fissures, hypertelorism, wide nasal bridge, malar flattening, small mouth with a thin upper lip, dysplastic ears with increased posterior angulation and a thick overfolded helix, proximally placed thumbs, broad square-shaped fingertips, single palmar crease on both hands, broad hallux, and overriding toes (Fig. 1). Additional anomalies were divergent concomitant strabismus, pectus excavatum, and bilateral flatfeet (pes planus). No seizures or known organ anomalies. Brain MRI at the age of 2 years did not show any pathological changes. A later evaluation of the pituitary gland was also normal. Ophthalmologic investigation revealed exotropia of the left eye. Visual acuity was reported 0.6 for the right eye and 0.7 for the left eye. He had clinically normal hearing and a definite response to $30 \mathrm{~dB}$ to behavioral observation audiometry screening. Hand radiology was not performed.

The patient had a younger sister who was a late walker at the age of 2 years, but the parents decided not to perform genetic testing.

The mother's psychomotor development was mildly delayed; she walked after the age of 1.5 years, had learning difficulties in school, and therefore attended a special school from 7 th grade on. She worked in child care as a nursery attendant. No dysmorphic features or anomalies were noted. Two spontaneous abortions during the first trimester were reported. 
Fig. 1. a-e Phenotype of the patient at the age of 9 years showing a triangular face, frontal bossing, prominent supraorbital ridges, downslanting palpebral fissures, hypertelorism, strabismus, a wide nasal bridge, malar flattening, small mouth with a thin upper lip (a-b), dysplastic ears with increased posterior angulation and a thick overfolded helix (c), proximally placed thumbs, broad square-shaped fingertips (d), broad hallux, and overriding toes (e).
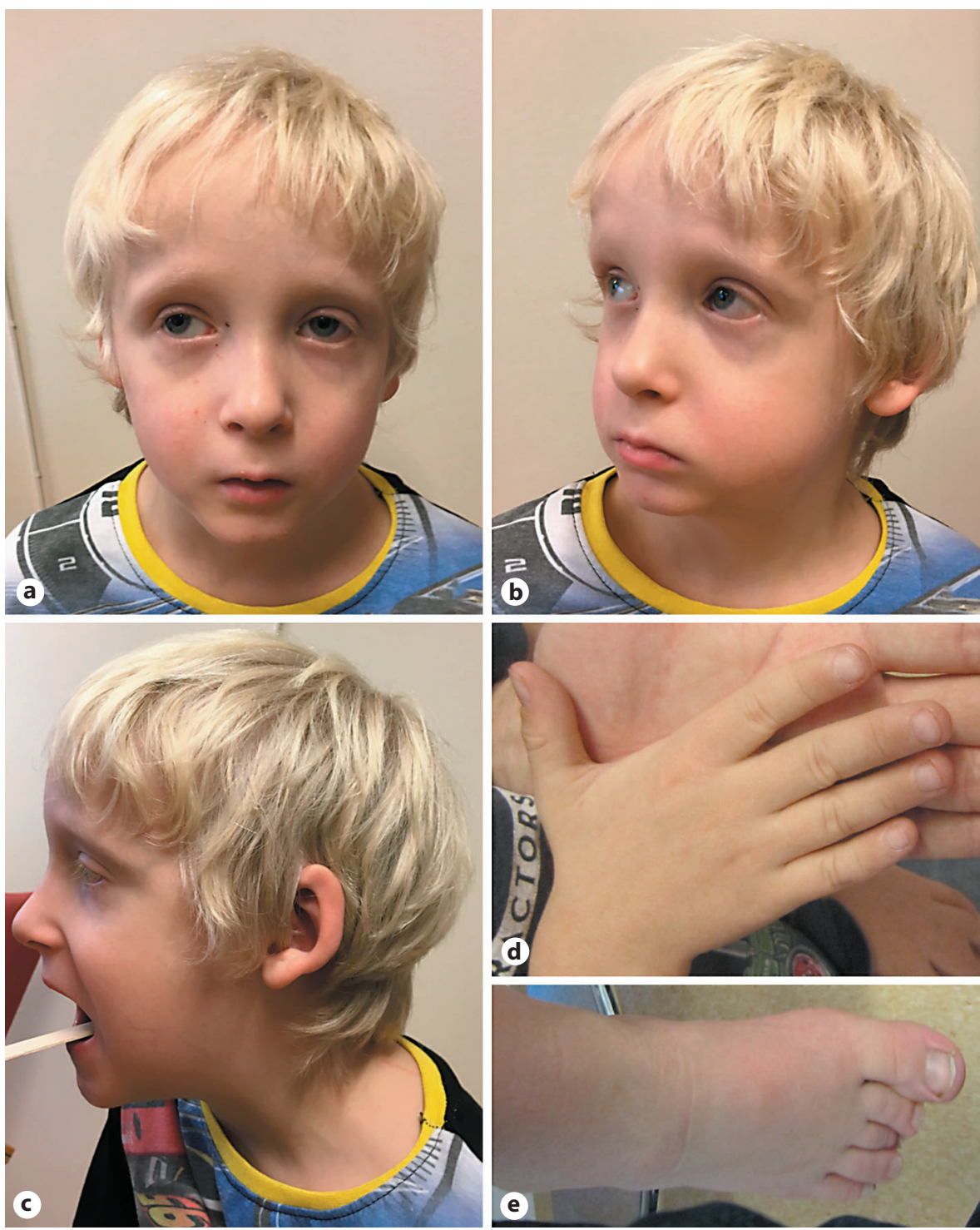

\section{Materials and Methods}

Previous genetic testing included SNP array and molecular analysis for myotonic dystrophy type 1, both with normal results.

Mass parallel sequencing analysis of disease-associated exon regions of the genome was performed on the patient and his parents (further referred to as trio-based exome sequencing) in the settings of a research project in order to screen for disease-causing gene variants.

DNA from patients and their parents were analyzed using TruSightOne assay (Illumina, first version), which includes 4,311 genes targeting Human Genome hg19. Paired-end sequencing $(2 \times$ $150 \mathrm{bp}$ ) was performed on a NextSeq (Illumina) instrument. The in-house bioinformatics pipeline included alignment with bwamem (version) and duplicate removal with picard (version); GATK (version) was used for realignment, recalibration, and vari- ant calling (Unified Genotyper). Vcf files were analyzed using Illumina Variant Studio (Illumina), where filtering and trio analysis were performed.

\section{Results}

Diagnostic trio-based exome sequence analyses revealed a novel hemizygous nonsense variant in the DLG3 gene (NM_021120.3), c.1720C>T; (p.Arg574*), in the patient and his mother. This was the only variant fulfilling XLID criteria. The variant was verified by Sanger sequencing (Fig. 2). X-inactivation analysis in the mother was noninformative due to homozygosity for the poly- 


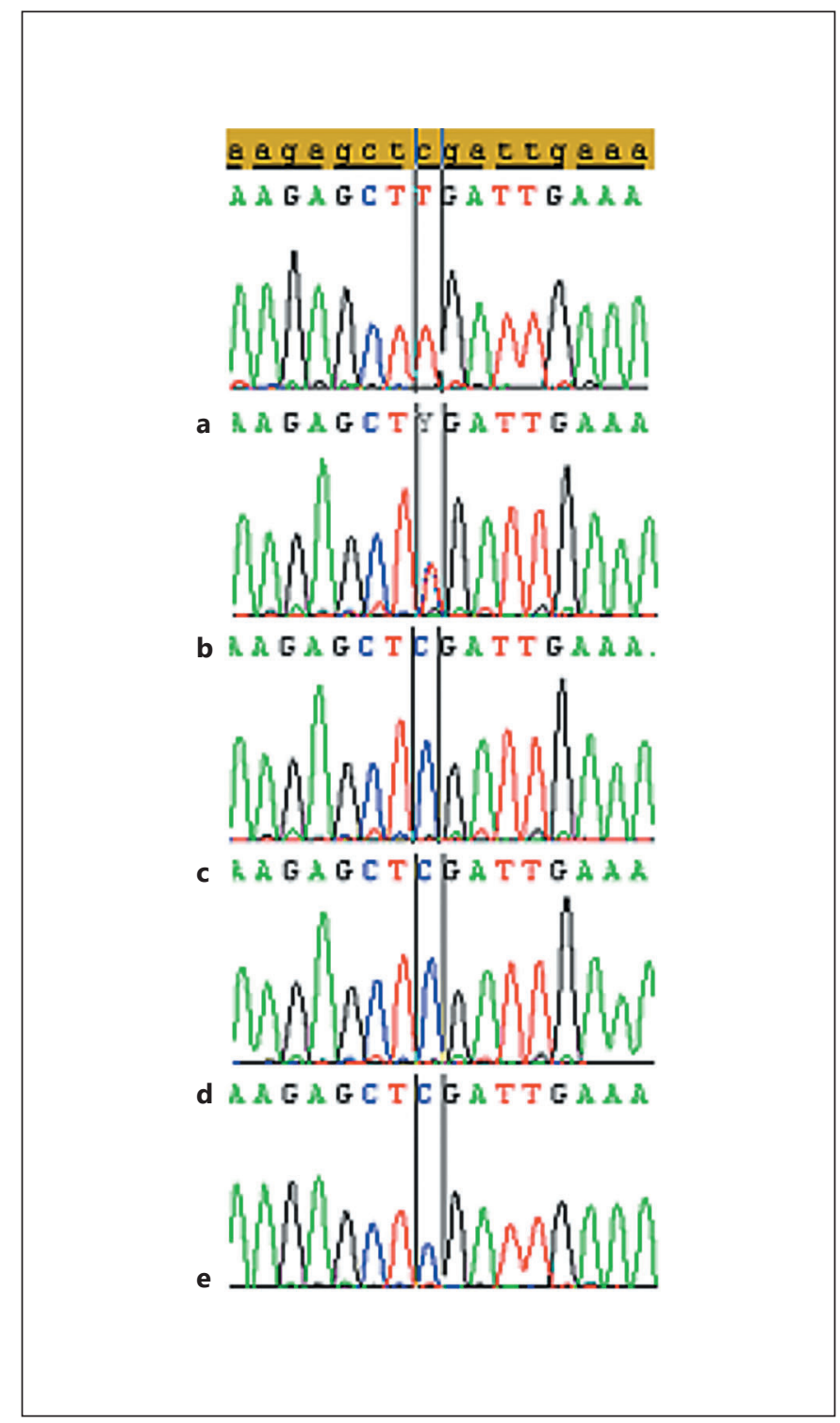

Fig. 2. Sanger sequencing electrophoregrams of the family for the nonsense DLG3 gene variant c.1720C>T; p.Arg574*. The reference sequence NM_021120.3 is indicated by a yellow bar. A nonsense gene variant was found as a hemizygous variant in the index patient (a) and as a heterozygous variant in his mother (b). Sanger sequencing of the father (c), maternal grandmother (d) and maternal grandfather $(\mathbf{e})$ at the same genome position complied with the reference.

morphic $A R$ repeat. Neither of the maternal grandparents showed this variant (Fig. 2). The younger sister of the patient has not been tested for this variant since the parents did not agree.

No other de novo or rare biallelic variants fulfilling the inclusion criteria were found in the patient.

\section{Discussion}

We report a family, a boy and his mother, with a novel nonsense gene variant of $D L G 3$, c.1720C $>\mathrm{T}$; p.Arg574*. The boy showed ID, hyperactive behavior, short stature due to GH deficiency, dysmorphic features (Fig. 1), left-sided eye exotropia, and pectus excavatum. The boy had no other major physical anomalies and had a normal brain MRI in consistence with the other patients described. The mother had learning disabilities and a borderline cognitive development and no other clinical features. Her disease-causing allele had arisen de novo. The $\mathrm{X}$-inactivation status could not be established as the test was noninformative.

Disease-causing mutations in the DLG3 gene were reported in 11 families so far, most of the affected patients are men with nonsyndromic ID, ranging from mild to severe [Tarpey et al., 2004; Zanni et al., 2010; Isrie et al., 2012; Philips et al., 2014; Tzschach et al., 2015; Kumar et al., 2016; Gieldon et al., 2017]. Some patients had additional symptoms including seizures, abnormal behavior, and strabismus [Tarpey et al., 2004; Zanni et al., 2010; Isrie et al., 2012; Philips et al., 2014; Tzschach et al., 2015; Kumar et al., 2016]. Mild facial dysmorphism was reported in only a few patients, but only occasional features seem to overlap with those of our patient. For example, a triangular face was observed in 2 of the previously described patients [Isrie et al., 2012; Gieldon et al., 2017]. Broad great toes were found in both the patient reported by Gieldon et al. [2017] as well as in the affected boy of this report. We observed quite specific facial dysmorphic features, not reported by others so far (Fig. 1), which included downslanting palpebral fissures. Philips et al. [2014] described upslanting palpebral fissures instead. Chest anomalies were seen in both the patients described by Philips et al. [2014] and in our patient. Kumar et al. [2016] reported a large multigenerational family where the affected males showed no facial dysmorphism, but some showed height below average, a few with a height below the 3 rd percentile. Short stature due to $\mathrm{GH}$ deficiency has not been mentioned in the previously reported patients and is unique to the boy presented here. One could speculate that the diverse clinical features observed in the patients with DLG3 nonsense mutations in addition to ID may be due to a diverse molecular consequence of those mutations, such as a truncated nonfunctional protein, nonsense-mediated mRNA decay, or deleterious gain of function of the resulting protein. 
This case report broadens the phenotypic spectrum of XLID caused by DLG3 nonsense variants. The dysmorphic features of the affected males may be more frequent than previously thought, although only occasionally recurrent. Short stature and chest anomalies were also found to be intermittently associated with DLG3 nonsyndromic XLID. Further observations and studies are needed to establish if the dysmorphic features are more frequent than thought in the patients with DLG3 nonsyndromic XLID. If true, DLG3 syndromic XLID may be a more appropriate term. The whole human exome has not been assessed with the present strategy. Thus, it is not possible from our data to exclude the action of other gene variants in the disease phenotype, which is a limitation of the study. However, the expanding use of whole-exome sequencing in the diagnostic of patients with ID is expected to add more to our knowledge of the phenotype of XLID due to DLG3 mutations.

\section{Acknowledgments}

We gratefully acknowledge the family for participating in this study. Special thanks and acknowledgments are also due to our colleague Dr. Jon Jonnasson for his valuable advices, discussion, and the revision of the manuscript.

\section{Statement of Ethics}

Ethics approval was provided by the Linköping University Ethics Committee, and written consent was obtained from both parents.

\section{Disclosure Statement}

The authors have no conflicts of interest to disclose.

\section{Funding Sources}

This work was funded by the Forskningsrådet Sydöstra Sverige (FORSS) as well as by ALF Grants, Region Östergötland (LIO440331).

\section{References}

Elias GM, Nicoll RA: Synaptic trafficking of glutamate receptors by MAGUK scaffolding proteins. Trends Cell Biol 17:343-352 (2007).

Funke L, Dakoji S, Bredt DS: Membrane-associated guanylate kinases regulate adhesion and plasticity at cell junctions. Annu Rev Biochem 74:219-245 (2005).

-Gieldon L, Mackenroth L, Betcheva-Krajcir E, Rump A, Beck-Wödl S, et al: Skewed X-inactivation in a family with $D L G 3$-associated Xlinked intellectual disability. Am J Med Genet 173:2545-2550 (2017).

-Isrie M, Froyen G, Devriendt K, de Ravel T, Fryns $J P$, et al: Sporadic male patients with intellectual disability: contribution of X-chromosome copy number variants. Eur J Med Genet 55:577-585 (2012).

Kennedy MB: Origin of PDZ (DHR, GLGF) domains. Trends Biochem Sci 20:350 (1995).
Kumar R, Ha T, Pham D, Shaw M, Mangelsdorf $\mathrm{M}$, et al: A non-coding variant in the $5^{\prime}$ UTR of DLG3 attenuates protein translation to cause non-syndromic intellectual disability. Eur J Hum Genet 24:1612-1616 (2016).

- Müller BM, Kistner U, Kindler S, Chung WJ, Kuhlendahl S, et al: SAP102, a novel postsynaptic protein that interacts with NMDA receptor complexes in vivo. Neuron 17:255-265 (1996).

Murata Y, Constantine-Paton M: Postsynaptic density scaffold SAP102 regulates cortical synapse development through EphB and PAK signaling pathway. J Neurosci 33:5040-5052 (2013).

Philips AK, Sirén A, Avela K, Somer M, Peippo M, et al: X-exome sequencing in Finnish families with intellectual disability - four novel mutations and two novel syndromic phenotypes. Orphanet J Rare Dis 9:49 (2014).

-Sheng M, Hoogenraad CC: The postsynaptic architecture of excitatory synapses: a more quantitative view. Annu Rev Biochem 76: 823-847 (2007).
Tarpey P, Parnau J, Blow M, Woffendin H, Bignell G, et al: Mutations in the DLG3 gene cause nonsyndromic X-linked mental retardation. Am J Hum Genet 75:318-324 (2004).

Tzschach A, Grasshoff U, Beck-Woedl S, Dufke C, Bauer C, et al: Next-generation sequencing in X-linked intellectual disability. Eur J Hum Genet 23:1513-1518 (2015).

-Won S, Levy JM, Nicoll RA, Roche KW: MAGUKs: multifaceted synaptic organizers. Curr Opin Neurobiol 43:94-101 (2017).

Zanni G, van Esch H, Bensalem A, Saillour Y, Poirier K, et al: A novel mutation in the $D L G 3$ gene encoding the synapse-associated protein 102 (SAP102) causes non-syndromic mental retardation. Neurogenetics 11:251255 (2010). 